\title{
FINANCIAMENTO DA SAÚDE: IMPASSES AINDA NÃO RESOLVIDOS
}

\author{
FUNDING HEALTH: UNSOLVED IMPASSES
}

Ana Luiza d'Ávila Viana ${ }^{1}$

Resumo O trabalho procura dialogar com os achados apresentados por Luciana Dias de Lima em seu texto sobre federalismo fiscal e financiamento descentralizado da saúde no Brasil, mediante a apresentação de resultados parciais e inéditos de um estudo, realizado no período 2006-2008, sobre estratégias de financiamento da atenção básica em municípios com mais de 100 mil habitantes do estado de São Paulo. O estudo envolveu a execução de dois módulos relacionados entre si: coleta e análise de dados secundários, buscando relacionar indicadores de financiamento da saúde no plano local com níveis de desempenho da atenção básica, e entrevistas semi-estruturadas com gestores de saúde de municípios selecionados. Os resultados mostram, de um lado, que os diferentes modelos de atenção básica existentes nos municípios, assim como os níveis diferenciados de eficácia e efetividade dos sistemas municipais de saúde, estão relacionados com o padrão de financiamento e gasto com saúde nesses municípios; de outro lado, que existem diversos aspectos que tendem a jogar papel decisivo na disponibilidade de recursos financeiros para o desenvolvimento da atenção básica, incluindo o comprometimento de recursos com serviços de média e alta complexidade e o alinhamento político do município com as demais esferas de governo.

Palavras-chave Sistema Único de Saúde; federalismo fiscal; financiamento; atenção básica.
Abstract The article seeks to dialogue with the discoveries presented by Luciana Dias de Lima in her article about fiscal federalism and decentralized funding of health in Brazil by presenting partial, unpublished results of a study carried out between 2006 and 2008 regarding basic care funding strategies in cities with more than 100,000 inhabitants in the State of São Paulo. The study involved two interrelated modules: secondary data collection and analysis, seeking to relate health funding indices on the local plan to basic care performance levels, and semi-structured interviews with health managers of selected cities. The results show, on one token, that the different basic care models that exist in the cities, as well as the differentiated levels of efficacy and effectiveness of the municipal health systems, are related to the health funding and expenditure standard in these cities; and, on the other, that there are several aspects that tend to play decisive roles in the availability of financial resources for the development of basic care, including committing resources with medium-complexity and high-complexity services and the city's political alignment with the remaining spheres of government.

Keywords Single Health System; fiscal federalism; funding; basic care. 
Como é de conhecimento daqueles que estudam a questão do financiamento da política de saúde brasileira, a insuficiência de recursos financeiros constitui um dos maiores obstáculos para a consolidação do Sistema Único de Saúde (SUS) no Brasil, evidenciando os limites para garantir a universalidade de acesso aos serviços públicos de saúde. Não se trata de uma questão trivial, na medida em que está relacionada com, pelo menos, dois problemas importantes. Em primeiro lugar, o financiamento da saúde, tal como definido pela Constituição Federal de 1988, envolve o compromisso das três esferas de governo (federal, estadual e municipal), o que supõe a existência de uma determinada estrutura de divisão de competências tributárias e de partilha intergovernamental de recursos, além de uma estrutura setorial de transferência de recursos entre as diferentes esferas de governo para o financiamento das ações e serviços descentralizados de saúde. Em segundo lugar, o montante de recursos federais depende das regras de partilha do chamado Orçamento da Seguridade Social (OSS) entre as três áreas que a integram - Saúde, Previdência e Assistência Social. Dessa for$\mathrm{ma}$, o estabelecimento de regras claras de participação de cada esfera governamental, assim como de regras justas e equilibradas de partilha no interior do OSS, é fundamental para garantir a disponibilidade de recursos e, com isso, a própria oferta de serviços de saúde - em quantidade e qualidade suficientes - à população.

Entretanto, não foi isso que ocorreu ao longo da chamada 'década expandida', termo utilizado por Luciana Dias de Lima em seu trabalho sobre federalismo fiscal e financiamento descentralizado da saúde para designar o período que vai de 1990 até os primeiros anos da década atual: o primeiro problema - regras de participação de cada esfera de governo - só foi parcialmente resolvido com a emenda constitucional n. ${ }^{\circ} 29$ (EC 29), aprovada em 2000, que estabeleceu percentuais mínimos de vinculação de recursos a serem investidos anualmente para financiar as ações e serviços públicos de saúde nos estados $(12 \%)$ e nos municípios $(15 \%)$, tomando por base a receita fiscal proveniente da arrecadação de impostos e das transferências governamentais nesses níveis de governo, e um percentual de aumento para o gasto federal segundo variações nominais do PIB; e o segundo problema, tão ou mais grave, encontra-se até hoje sem definição, e constitui alvo e elemento preferencial da política fiscal, na medida em que parte dos recursos do OSS é usada tanto para cobrir despesas correntes da União quanto para formar fundos de estabilização fiscal.

O trabalho de Luciana Dias de Lima consegue mostrar, de modo apropriado, a existência de aspectos-chave do federalismo fiscal e setorial que impactam no financiamento da saúde. De modo geral, pode-se verificar que a relação entre os dois mecanismos de financiamento das esferas subnacionais expressam o embate entre o federalismo fiscal e o setorial: os crité- 
rios da EC 29 (e das receitas fiscais vinculadas) 'não conversam' com os critérios utilizados pelo governo federal para as transferências do SUS, ou seja, os critérios não são definidos um em relação ao outro e, por isso, não resolvem os conflitos verticais nem horizontais. O principal resultado a ser mencionado é que, em ambos os casos, os municípios foram os que mais se beneficiaram do processo de descentralização de recursos; entretanto, as desigualdades entre eles não foram solucionadas, nem através das transferências fiscais, nem através da vinculação de recursos ou das transferências do SUS.

Em trabalho anterior (Lima, 2007), a autora já havia demonstrado que as principais fontes de recurso dos municípios para o financiamento das ações e serviços de saúde são oriundas das receitas próprias, dada pela base vinculável, formada pelos impostos diretamente arrecadados e pelas transferências constitucionais e legais, de modo que as transferências SUS desempenham, de modo geral, um papel secundário. Mas esse comportamento não é homogêneo quando os municípios são analisados em função do porte populacional: no caso das receitas vinculadas à EC 29, a importância é inversamente proporcional ao tamanho dos municípios, o que é reflexo da importância do Fundo de Participação dos Municípios (FPM) nos municípios de menor porte, ao mesmo tempo em que a arrecadação própria e as transferências devolutivas/compensatórias são mais importantes nos municípios de maior porte e nas capitais; com relação às transferências SUS, acontece exatamente o oposto - as cidades maiores são as mais beneficiadas com o repasse de recursos federais para o financiamento da saúde. Para complicar ainda mais a situação, a despeito do maior esforço de gasto dos estados e municípios, os constrangimentos impostos pela política econômica às finanças estaduais e municipais dificultaram ou impediram o exercício pleno de suas atribuições no SUS, limitando a ocorrência de um aumento mais expressivo do gasto local em saúde, uma maior autonomia perante os recursos federais e a aplicação de um volume mais elevado de recursos próprios no financiamento da saúde.

Estudo recente desenvolvido pelo Departamento de Medicina Preventiva da Faculdade de Medicina da Universidade de São Paulo sobre o financiamento da atenção básica em municípios com população superior a 100 mil habitantes do estado de São Paulo (Viana, 2008) produziu resultados que permitem aprofundar e discutir alguns aspectos levantados pelo trabalho de Luciana Dias de Lima. Apresentamos, a seguir, um resumo dos principais achados obtidos em dois módulos do estudo - análise de dados secundários e entrevistas semi-estruturadas com gestores municipais de saúde. 


\section{Relações entre financiamento e atenção básica}

Os dados obtidos pela Pesquisa de Avaliação do Proesf (Consórcio Medicina USP, 2007) possibilitaram construir indicadores mostrando o modelo predominante de atenção básica existente nos municípios, a eficácia da gestão e a efetividade dos sistemas municipais de saúde quanto à integralidade do serviço e ao acesso universal às ações de atenção básica. Com base nesses indicadores, o estudo procurou analisar a relação entre o padrão de financiamento e gasto municipal com saúde e a situação da atenção básica nos municípios paulistas com mais de 100 mil habitantes. Para caracterizar o padrão de financiamento e gasto municipal com saúde, foram utilizados os seguintes indicadores: capacidade fiscal do município, dada pela soma das receitas de impostos e transferências constitucionais por habitante/ano; despesa total com saúde por habitante/ano; percentual de recursos próprios aplicados na saúde; e percentual de transferências SUS sobre a despesa total com saúde. A fonte utilizada foi o Sistema de Informação sobre Orçamento Público em Saúde (Siops) [série histórica], e os dados se referem ao ano de 2006.

Os resultados da análise em relação aos indicadores de desempenho da atenção básica mostraram que o grupo de municípios cujo modelo enfatiza a estratégia de saúde da família apresenta menor capacidade fiscal, menor despesa total com saúde, menor percentual de recursos próprios aplicados na saúde e maior percentual de transferências SUS. Já os municípios do grupo centrado na atenção básica em unidades públicas apresentam maior capacidade fiscal, maior despesa total com saúde e maior percentual de recursos próprios aplicados na saúde. E os municípios do grupo com elevada participação de procedimentos especializados na produção ambulatorial destacam-se por apresentar capacidade fiscal acima da média e percentual de transferências SUS abaixo da média.

No caso da eficácia da gestão, percebe-se uma associação positiva entre a eficácia da gestão municipal e os indicadores de financiamento e gasto com saúde, ou seja, municípios com níveis mais elevados de organização e avaliação apresentam indicadores mais favoráveis de financiamento. Tais resultados sugerem que o padrão de financiamento e gasto municipal com saúde tende a exercer influência importante nas capacidades de organização e de avaliação dos municípios brasileiros na área da saúde.

Em relação à efetividade, verifica-se a existência de três situações: a) municípios que não possuem integralidade nem acesso universal apresentam menor despesa total com saúde por habitante/ano, percentual mais baixo de recursos próprios aplicados na saúde e de transferências SUS sobre a despesa total com saúde; b) municípios que possuem integralidade ou acesso universal apresentam maior capacidade fiscal, maior despesa total 
com saúde e percentual mais alto de recursos próprios aplicados na saúde; e c) municípios que possuem integralidade e acesso universal apresentam menor capacidade fiscal e maior participação das transferências SUS na despesa total com saúde (Tabela 1).

Tabela 1

Classificação dos municípios em função do cruzamento dos indicadores de desemprego da atenção básica e dos indicadores de financiamento e gasto

\begin{tabular}{|c|c|c|c|c|}
\hline \multirow{2}{*}{$\begin{array}{l}\text { Financiameto e } \\
\text { gasto com saúde }\end{array}$} & \multirow[t]{2}{*}{ Valores } & \multicolumn{3}{|c|}{ Desempenho da Atenção Básica } \\
\hline & & Modelo AB & Eficácia & Efetividade \\
\hline \multirow[t]{2}{*}{ Capacidade fiscal } & Maior & $\begin{array}{l}\text { Centrado na } A B \text { em } \\
\text { unidades públicas }\end{array}$ & $\begin{array}{l}\text { Capacidade de } \\
\text { organização baixa e } \\
\text { de avaliação alta }\end{array}$ & $\begin{array}{l}\text { Integralidade } \\
\text { ou acesso }\end{array}$ \\
\hline & Menor & $\begin{array}{l}\text { Centrado na } A B \text { com } \\
\text { ênfase no PSF }\end{array}$ & $\begin{array}{l}\text { Capacidade de } \\
\text { organização alta e } \\
\text { de avaliação baixa }\end{array}$ & $\begin{array}{l}\text { Integralidade } \\
\text { e acesso }\end{array}$ \\
\hline \multirow[t]{2}{*}{$\begin{array}{l}\text { Despesa total com } \\
\text { saúde }\end{array}$} & Maior & $\begin{array}{l}\text { Centrado na } A B \text { em } \\
\text { unidades públicas }\end{array}$ & $\begin{array}{l}\text { Capacidade de } \\
\text { organização alta e } \\
\text { de avaliação baixa }\end{array}$ & $\begin{array}{l}\text { Integralidade } \\
\text { ou acesso }\end{array}$ \\
\hline & Menor & $\begin{array}{l}\text { Centrado na } A B \text { com } \\
\text { ênfase no PSF }\end{array}$ & $\begin{array}{l}\text { Capacidade de } \\
\text { organização alta e } \\
\text { avaliação baixa }\end{array}$ & $\begin{array}{l}\text { Não tem } \\
\text { integralidade } \\
\text { nem acesso }\end{array}$ \\
\hline \multirow[t]{2}{*}{$\begin{array}{l}\text { Dependência do } \\
\text { SUS Federal }\end{array}$} & Maior & $\begin{array}{l}\text { Centrado na } A B \text { com } \\
\text { ênfase no PSF }\end{array}$ & $\begin{array}{l}\text { Capacidade de } \\
\text { organização alta e } \\
\text { de avaliação baixa }\end{array}$ & $\begin{array}{l}\text { Integralidade } \\
\text { e acesso }\end{array}$ \\
\hline & Menor & $\begin{array}{l}\text { Centrado na } A B \text { em } \\
\text { unidades públicas }\end{array}$ & $\begin{array}{l}\text { Capacidade de } \\
\text { organização baixa e } \\
\text { de avaliação baixa }\end{array}$ & $\begin{array}{l}\text { Não tem } \\
\text { integralidade } \\
\text { nem acesso }\end{array}$ \\
\hline \multirow[t]{2}{*}{$\begin{array}{l}\text { Cumprimento da } \\
\text { EC } 29\end{array}$} & Maior & $\begin{array}{l}\text { Centrado na } A B \text { em } \\
\text { unidades públicas }\end{array}$ & $\begin{array}{l}\text { Capacidade de } \\
\text { organização alta e } \\
\text { de avaliação alta }\end{array}$ & $\begin{array}{l}\text { Integralidade } \\
\text { ou acesso }\end{array}$ \\
\hline & Menor & $\begin{array}{l}\text { Centrado na } A B \text { com } \\
\text { ênfase no PSF }\end{array}$ & $\begin{array}{l}\text { Capacidade de } \\
\text { organização baixa e } \\
\text { de avaliação baixa }\end{array}$ & $\begin{array}{l}\text { Não tem } \\
\text { integralidade } \\
\text { nem acesso }\end{array}$ \\
\hline
\end{tabular}

Fonte: Siops; Consórcio Medicina USP (2007-2008).

O principal achado desses cruzamentos é que o padrão de financiamento e gasto municipal com saúde está estreitamente relacionado com o desempenho da atenção básica no universo de municípios estudados. De fato, 
maior capacidade fiscal, maior despesa total com saúde e maior percentual de recursos próprios aplicados na saúde são indicadores que estão associados a um grupo selecionado de municípios, a saber: aqueles que possuem modelo de atenção básica centrado em unidades púbicas, níveis elevados de organização e avaliação, assim como integralidade e acesso. Por outro lado, municípios cujo modelo de atenção básica enfatiza o PSF possuem níveis mais baixos de organização e avaliação, não têm integralidade nem acesso universal, apresentam menor capacidade fiscal, menor despesa total com saúde, maior dependência de recursos federais e destinam menos recursos próprios para a saúde.

\section{Questões relacionadas ao financiamento na percepção de gestores municipais de saúde}

A realização de entrevistas com gestores de saúde de sete municípios paulistas com mais de 100 mil habitantes, representativos de grupos homogêneos segundo características relacionadas com a situação socioeconômica do município e com o grau de complexidade da produção ambulatorial, assim como de diferentes portes populacionais, possibilitou coletar um conjunto importante de informações relacionadas à questão do financiamento da saúde no plano local, com ênfase nas ações de atenção básica (ver Tabela 2, na página ao lado).

Um primeiro aspecto ressaltado por vários gestores é a pouca disponibilidade de recursos financeiros para serem aplicados na atenção básica, principalmente por aqueles que precisam comprometer parcela importante dos recursos com procedimentos de atenção especializada, cuja oferta é insuficiente para atender a demanda atualmente existente nesses municípios. Entretanto, como reconhecem os gestores, essa demanda explosiva por serviços especializados pode ser um indicador importante de que a atenção básica não está conseguindo resolver os problemas de saúde mais simples da população, ao mesmo tempo em que há falta de coordenação entre os diferentes níveis de assistência.

No que diz respeito ao repasse de recursos financeiros para o SUS municipal, a participação das transferências SUS na despesa total com saúde nesses municípios varia de $11 \%$ em Cotia até $43 \%$ em São Carlos, o que sugere a existência de diferentes graus de dependência em relação aos recursos repassados pelo Ministério da Saúde (MS). De modo geral, é possível classificar esses municípios em dois grupos - um primeiro grupo, formado pelos municípios de Cotia, Cubatão e Presidente Prudente, cuja participação das transferências SUS na despesa total com saúde é relativamente baixa (até $20 \%$ ), e um segundo grupo, formado pelos municípios de Santo André, 
Municípios selecionados para realização da pesquisa de campo, por porte populacional, segundo grupos homogêneos de municípios

\begin{tabular}{|c|c|c|c|c|}
\hline \multirow{2}{*}{$\begin{array}{l}\text { Grupos homogêneos de } \\
\text { municípios (clusters) }\end{array}$} & \multicolumn{4}{|c|}{ Porte populacional (habitantes) } \\
\hline & $100-200 \mathrm{mil}$ & $200-500 \mathrm{mil}$ & 500-1 milhão & $>1$ milhão \\
\hline $\begin{array}{l}\text { 1- Baixa complexidade e } \\
\text { indicadores sociais menos } \\
\text { favoráveis }\end{array}$ & Cotia & - & - & - \\
\hline $\begin{array}{l}\text { 2- Média complexidade e } \\
\text { indicadores sociais menos } \\
\text { favoráveis }\end{array}$ & Cubatão & - & Santo André & - \\
\hline $\begin{array}{l}\text { 3- Alta complexidade e } \\
\text { indicadores sociais menos } \\
\text { favoráveis }\end{array}$ & - & Santos & - & - \\
\hline $\begin{array}{l}\text { 4-Baixa complexidade e } \\
\text { indicadores sociais mais } \\
\text { favoráveis }\end{array}$ & - & São Carlos & - & - \\
\hline $\begin{array}{l}\text { 5- Média complexidade e } \\
\text { indicadores sociais mais } \\
\text { favoráveis }\end{array}$ & $\begin{array}{l}\text { Presidente } \\
\text { Prudente }\end{array}$ & - & - & - \\
\hline $\begin{array}{l}\text { 6- Alta complexidade e } \\
\text { indicadores sociais mais } \\
\text { favoráveis }\end{array}$ & - & - & - & Campinas \\
\hline
\end{tabular}

Fonte: $\mathrm{O}$ autor.

Campinas, Santos e São Carlos, que apresentam maior dependência dos recursos provenientes do Ministério da Saúde (acima de 30\%).

Também é interessante notar que esses dados guardam alguma relação com o alinhamento político dos prefeitos desses municípios com o executivo federal. De fato, três dos quatro municípios do segundo grupo possuem prefeitos que são do mesmo partido político (PT) do presidente da República - Santo André e São Carlos - ou de partidos aliados (PDT) no plano local - Campinas. Por outro lado, dois dos três municípios do primeiro grupo Cotia e Presidente Prudente - são administrados por prefeitos que pertencem ao principal partido de oposição (PSDB). Essa situação sugere que, possivelmente, aspectos relacionados à esfera política tendem a influenciar a natureza da relação entre as secretarias municipais de saúde e o Ministério da Saúde, no que diz respeito ao repasse de recursos financeiros. De fato, a questão da articulação política do município com o governo federal e com o governo estadual foi um aspecto ressaltado por vários gestores de saúde durante as entrevistas. O que se observa, pelos depoimentos coletados, é que 
os municípios politicamente alinhados com uma ou outra esfera de governo tendem a ter suas demandas atendidas com mais facilidade, ou seja, a filiação político-partidária do prefeito constitui um fator que faz diferen-ça para a obtenção de mais recursos financeiros para a área da saúde (Gráfico 1).

Participação das transferências SUS na despesa total com saúde - 2007

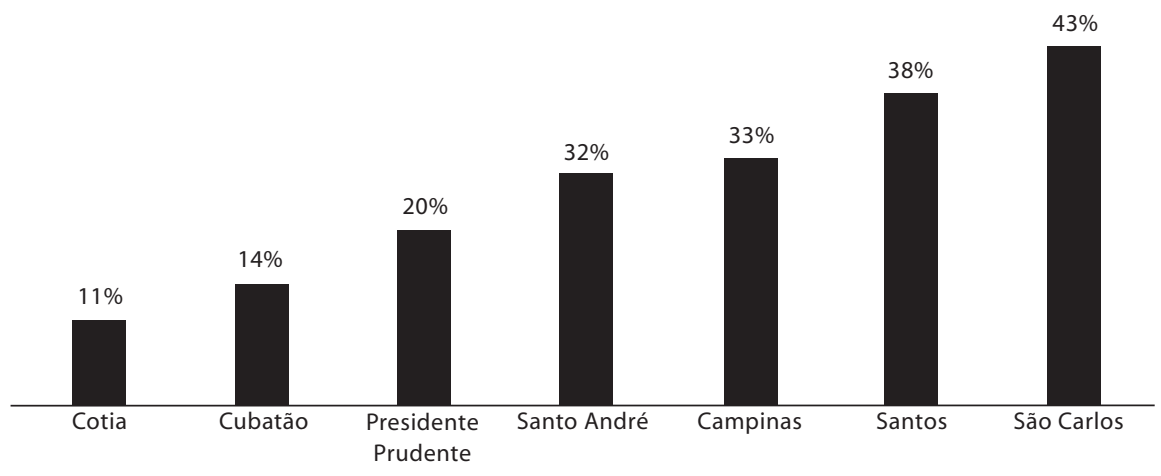

Fonte: Siops, Ministério da Saúde.

A maior parte dos recursos repassados pelo Ministério da Saúde aos municípios selecionados relaciona-se com o desenvolvimento de ações e serviços de média e alta complexidade, chegando a representar $81 \%$ do total no município de Santos. Duas exceções chamam a atenção - Cotia e Presidente Prudente; nesses dois municípios, prevalecem repasses enquadrados no bloco de atenção básica, sendo que o percentual relativo a média e alta complexidade é bastante baixo. Não por acaso, esses dois municípios passaram por crises recentes na oferta de serviços de média e de alta complexidade, com reflexos para a organização da atenção básica, sendo que a solução adotada foi a transferência de parte ou da totalidade da gestão da média e alta complexidade para o governo estadual.

A distribuição de recursos entre Piso de Atenção Básica (PAB) fixo e PAB variável é outro aspecto que merece ser destacado, na medida em que, dentro do bloco de atenção básica, o valor relativo ao PAB fixo é predominante em todos os municípios, com exceção de Cubatão, onde o valor do PAB variável é superior. Também não por acaso, o gestor desse município foi o que mais enfatizou a importância da estratégia de saúde da família como modelo de organização da atenção básica, sendo o único a explicitar o desejo de estender o programa para todo o município. 
De modo geral, os gestores de saúde entrevistados consideram importantes os recursos transferidos pelo Ministério da Saúde para o SUS municipal, na medida em que complementam os recursos aplicados pelo Tesouro municipal. Com relação aos recursos repassados pelo Ministério da Saúde para o desenvolvimento de ações de atenção básica, os gestores reconhecem a importância da criação do Piso de Atenção Básica e da forma - regular e automática - como ele é repassado aos municípios. Apesar desse avanço na forma de repasse dos recursos para a atenção básica, os gestores apontam que os recursos são insuficientes para o desenvolvimento das ações, inclusive para o custeio das equipes que trabalham na estratégia de saúde da família.

Ao contrário dos repasses regulares e automáticos que são transferidos fundo a fundo, destinados principalmente para custeio dos sistemas de saúde locais, parcela importante dos recursos provenientes da celebração de convênios entre o Ministério da Saúde e os municípios está relacionada, de um lado, com a construção, manutenção e reforma de unidades de saúde ambulatoriais e hospitalares e, de outro, com a aquisição de equipamento e material permanente. De fato, $95 \%$ dos recursos associados a convênios celebrados entre o MS e os sete municípios visitados no período 2005-2007 (Gráfico 2) dizem respeito ao desenvolvimento desses dois conjuntos de ações. Embora esses recursos sejam considerados importantes pelos gestores de saúde, seu caráter temporário é visto com preocupação, na medida em que são vinculados ao desenvolvimento de projetos específicos, com começo, meio e fim.

\section{Gráfico 2}

Distribuição percentual dos convênios celebrados entre o MS e os municípios visitados no período 2005-2007

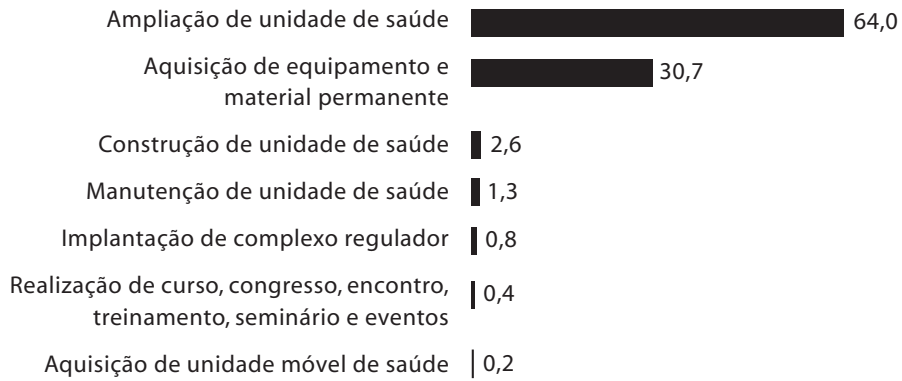

Fonte: Fundo Nacional de Saúde (Funasa).

Nota: Inclui somente convênios já pagos ou em andamento (pago parcialmente).

Os gestores de saúde fizeram comentários sobre os critérios utilizados pelo Ministério da Saúde para o repasse de recursos financeiros aos fundos 
municipais de saúde. De modo geral, como mostram os depoimentos coletados durante a pesquisa de campo, a adoção do critério per capita para cálculo do montante a ser repassado a cada município e a criação dos blocos de financiamento foram aspectos valorizados pelos gestores de saúde. Por outro lado, a falta de mobilidade dos recursos entre os diferentes blocos de financiamento foi objeto de crítica por parte dos gestores, que consideram equivocada a estratégia do Ministério da Saúde de induzir o desenvolvimento de programas de saúde por dinheiro. Além disso, defendem a idéia de que, no longo prazo, o Ministério da Saúde acabe com os blocos de financiamento e adote como critério de repasse os resultados apresentados pelo município na aplicação dos recursos, premiando aqueles que conseguem alcançar resultados positivos e punindo aqueles que não atingem as metas planejadas.

Outro tema bastante comentado pelos gestores de saúde dos municípios visitados foi o baixo nível de participação do governo estadual no financiamento do sistema de saúde local. Essa constatação foi feita por todos os gestores entrevistados, que destacaram que o estado praticamente não transfere recursos financeiros diretamente aos municípios. Por outro lado, eles reconhecem que a secretaria estadual de saúde, com sua rede de atendimento especializado, presta um serviço importante para diagnóstico, tratamento e recuperação da saúde da população residente nesses municípios. O problema, segundo esses gestores, é a dificuldade de mensurar a dimensão dessa participação. Ao mesmo tempo, apontam que o governo estadual não tem sido capaz de exercer seu papel de coordenação e regulação do sistema de saúde de forma satisfatória, fazendo com que, muitas vezes, haja competição com os municípios - ao invés de complementaridade - na oferta de serviços.

Com relação à determinação do montante de recursos próprios a serem aplicados na saúde, diversos fatores foram destacados pelos gestores de saúde. No plano mais geral, essa determinação tem a ver com as diretrizes, os objetivos e as prioridades definidas no Plano Municipal de Saúde, que constitui o documento formal da política de saúde no município, e com os programas e as ações incluídas no Plano Plurianual (PPA) de cada município. Entretanto, apesar de todo o planejamento dos programas e ações na área da saúde, foi mencionado que o orçamento da saúde tende a apresentar muita variação de um ano para o outro, em função de acontecimentos imprevistos, como, por exemplo, aumentos inesperados dos preços de insumos ou necessidade de execução de obras que não haviam sido planejadas. Outro aspecto destacado é o papel desempenhado pelo gestor de saúde e sua relação com o gestor da área de finanças, bem como seu alinhamento com o prefeito do município.

Apesar de os municípios visitados apresentarem percentuais diferentes de participação da receita própria aplicada em saúde, os gestores de saúde 
possuem opinião semelhante a respeito da compatibilidade entre o montante de recursos próprios aplicados na saúde e a capacidade fiscal do município. Essa opinião é a de que há pouco espaço para que o município aumente essa participação e, portanto, a quantidade de recursos próprios aplicados na saúde. De um lado, os gestores destacaram que a capacidade de arrecadação fiscal dos municípios encontra-se praticamente no limite, com pouca possibilidade de aumento, inclusive em virtude do tamanho da economia informal, que não recolhe tributos, e da sonegação fiscal; de outro, sublinharam que direcionar mais recursos próprios para a saúde significa retirar recursos de outras áreas que são igualmente importantes para o desenvolvimento do município. Além disso, mencionaram a necessidade de aperfeiçoar a administração do gasto em saúde, para que a aplicação dos recursos disponíveis possa produzir mais resultados. Por fim, chamaram a atenção para o fato de que a ampliação de recursos para a saúde é uma discussão que deve ser feita por toda a sociedade que contribui para a manutenção do sistema de saúde no plano local, via pagamento de tributos, e não somente pelos gestores.

A questão da autonomia na utilização dos recursos disponíveis no Fundo Municipal de Saúde mostrou que os municípios vivenciam realidades diferenciadas, sendo possível identificar duas situações de acordo com o nível de autonomia do gestor na utilização dos recursos para a saúde. A primeira situação - mais comum e que engloba seis dos sete municípios visitados - é de municípios com Fundo Municipal de Saúde localizado na secretaria de saúde, sendo que a movimentação financeira das contas é feita pelo gestor de saúde (secretário de saúde ou outro profissional responsável pelo fundo), que não precisa de autorização prévia de nenhuma outra instância externa à secretaria de saúde para utilizar os recursos disponíveis de acordo com a programação de despesas. A segunda situação é representada somente pelo município de Cubatão, onde o gestor de saúde não é o responsável direto pela gestão do Fundo Municipal de Saúde, que está localizado em outra área da prefeitura - Secretaria de Administração e Planejamento; dessa forma, a administração dos recursos disponíveis para a saúde não é feita pelo gestor de saúde, mas por outra área, de forma centralizada. Essa situação, segundo o gestor desse município, representa um aspecto que, tecnicamente, atrapalha o bom funcionamento das ações executadas pela secretaria de saúde, em função da morosidade no processo de execução financeira.

Finalmente, os gestores entrevistados manifestaram opiniões divergentes sobre a emenda constitucional $n .^{\circ} 29$, que estabelece o percentual mínimo de vinculação de recursos para o financiamento das ações e serviços públicos de saúde em cada esfera de governo. Embora haja a compreensão de que a EC 29 conseguiu direcionar mais recursos para a saúde, diversos 
gestores apontaram que a falta de regulamentação da emenda é um dos fatores que possibilitam seu descumprimento na prática. Ao mesmo tempo, alguns gestores criticaram os parâmetros utilizados para vinculação dos recursos.

\section{Pouca luz no fim do túnel}

Tanto o trabalho desenvolvido por Luciana Dias de Lima quanto o estudo coordenado por Viana apresentam resultados que merecem ser analisados e discutidos não só pelos pesquisadores interessados no tema, mas, principalmente, por aqueles responsáveis pela formulação e implementação de políticas públicas na área da saúde, na medida em que levantam um conjunto importante de questões que confirmam a fragilidade dos mecanismos de financiamento da política de saúde no Brasil. Dois episódios recentes mostram que as discussões em torno dessas questões estão longe de encontrar uma solução definitiva.

Em primeiro lugar, a não prorrogação da Contribuição Provisória sobre Movimentação Financeira (CPMF), em dezembro de 2007, representou o fim de uma das principais fontes de recurso do governo federal para o financiamento da saúde, o que tende a comprometer a capacidade de execução das políticas de investimento definidas pelo Ministério da Saúde. De fato, em 2007, a arrecadação da CPMF foi de R\$ 36,5 bilhões. Entretanto, é preciso destacar que os recursos arrecadados pela cobrança da CPMF foram de uso exclusivo da saúde apenas nos dois primeiros anos (1997 e 1998): em 1999, parcela dos recursos passou a ser direcionada para financiamento da Previdência Social e, em 2001, outra parcela passou a formar o Fundo de Combate e Erradicação da Pobreza. Por fim, é preciso lembrar que percentual importante da CPMF, assim como das demais contribuições sociais, não foi aplicado em nenhuma das três áreas sociais, ficando disponível no caixa do Tesouro para ser utilizado em outras áreas, de modo que a parcela efetivamente repassada para a saúde, nos últimos anos, foi de aproximadamente $40 \%$ do total arrecadado. O mesmo poderá acontecer com a Contribuição Social da Saúde (CSS), cuja proposta de criação foi encaminhada pelo Executivo Federal no primeiro semestre de 2008 ao Congresso Nacional como forma de compensar as perdas de arrecadação decorrentes do fim da cobrança da CPMF.

Em segundo lugar, a regulamentação da EC 29 encontra-se em tramitação no Congresso Nacional desde 2003, sinalizando claramente a falta de empenho dos poderes Executivo e Legislativo no encaminhamento da matéria. Conforme destacado pelo mais recente Boletim de Politicas Sociais do Ipea (Ipea, 2008), três pontos têm sido objeto de discussão e preocupação 
quase contínua desde a aprovação da emenda: a) a falta de uma definição do que deve ser considerado como ações e serviços públicos de saúde para efeitos da vinculação de recursos; b) o volume de recursos, que ainda é considerado insuficiente, seja para fazer frente às necessidades do setor, seja em comparação com outros sistemas de saúde que buscam a universalidade e integralidade da atenção (como é preconizado pelo SUS); e c) a utilização de dois critérios de vinculação - para estados e municípios, os recursos a serem aplicados na área de saúde foram vinculados a um percentual da receita corrente; para a União, a vinculação está relacionada ao crescimento do Produto Interno Bruto (PIB). Todos esses pontos, vale lembrar, fazem parte do rol de questões a serem resolvidas por lei complementar. Além disso, a demora em aprovar a regulamentação da EC 29 significa, de um lado, manter as atuais possibilidades de descumprimento da vinculação e, de outro, impedir que mais recursos do governo federal sejam aplicados na saúde, caso não haja alteração na regra de vinculação da União - da variação nominal do PIB para $10 \%$ das receitas brutas. De acordo com estimativas apresentadas por Dain (2007), a saúde deixou de contar com quase $\mathrm{R} \$ 80$ bilhões de recursos provenientes da União, no período 2000-2006, por conta da atual sistemática de cálculo. Estima-se ainda que a aprovação do projeto de lei que regulamenta a EC 29 implique aporte adicional de R\$ 23 bilhões para a saúde até 2011.

\section{Nota}

1 Professora do Departamento de Medicina Preventiva da Faculdade de Medicina da Universidade de São Paulo (USP). Doutora em Ciência Econômica pela Universidade Estadual de Campinas (Unicamp). <anaviana@usp.br>

Correspondência: Universidade de São Paulo, Faculdade de Medicina, Departamento de Medicina Preventiva, Avenida Doutor Arnaldo, 455, São Paulo, Brasil, CEP 01246-903.

\section{Referências}

CONSÓRCIO MEDICINA USP. Inquérito com usuários e profissionais, percepção dos gestores e estudos sobre o trabalho do PSF. São Paulo: Centro de Estudos de Cultura Contemporânea, Consórcio Medicina USP,
2007. Cadernos de Atenção Básica: Estudos Analíticos, 3.

DAIN, Sulamis. Os vários mundos do financiamento da Saúde no Brasil: uma tentativa 
de integração. Ciência \& Saúde Coletiva, Rio de Janeiro, v. 12, 2007, p. 1851-1864. Suplemento.

GERSCHMAN, Silvia; VIANA, Ana Luiza d'Ávila. Descentralização e desigualdades regionais em tempos de hegemonia liberal. In: LIMA, Nísia Trindade et al. Saúde e democracia: história e perspectivas do SUS. Rio de Janeiro: Editora Fiocruz, 2005.

IPEA. Boletim de políticas sociais: acompanhamento e análise, Brasília, n. 15, mar. 2008, p. 73-95. Disponível em: <www.ipea. gov.br/sites/000/2/publicacoes/bpsociais/bp s_15/16_completo.pdf $>$. Acesso em: 26 maio 2008 .

LIMA, Luciana Dias de. Federalismo, relações fiscais e financiamento do Sistema Único de Saúde: a distribuição de receitas vinculadas à saúde nos orçamentos municipais e estaduais. Rio de Janeiro: Museu da República; 2007.

VIANA, Ana Luiza d'Ávila (Coord). Estratégias de financiamento para organização e fortalecimento da atenção básica nos grandes centros urbanos de São Paulo. [Relatório de Pesquisa]. São Paulo: Universidade de São Paulo, 2008. 











